Annuaire du Collège de France 2008-2009

\title{
Analyse et géométrie
}

\section{Alain Connes}

\section{OpenEdition}

Journals

Édition électronique

URL : https://journals.openedition.org/annuaire-cdf/230

DOI : 10.4000/annuaire-cdf.230

ISBN : 978-2-7226-0138-3

ISSN : 2109-9227

Éditeur

Collège de France

Édition imprimée

Date de publication : 1 mars 2010

Pagination : 81-91

ISBN : 978-2-7226-0083-6

ISSN : 0069-5580

Référence électronique

Alain Connes, «Analyse et géométrie », L'annuaire du Collège de France [En ligne], 109 | 2010, mis en ligne le 24 juin 2010, consulté le 22 août 2022. URL : http://journals.openedition.org/annuaire-cdf/230 ; DOI : https://doi.org/10.4000/annuaire-cdf.230 


\section{Analyse et géométrie}

M. Alain Connes, membre de l'Institut

(Académie des sciences), professeur

\section{Le Monoïde des Classes d'Adèles}

\section{INTRODUCTION}

Mon cours cette année portait sur les résultats récents obtenus en collaboration avec C. Consani. Nous avons obtenu dans [3] la formule (1) ci-dessous pour la fonction de comptage $N(q), q \in[1, \infty)$ associée par C. Soulé à la courbe hypothétique $C=\overline{\mathrm{Spec} Z}$ sur $\mathbb{F}_{1}$ dont la fonction zêta $\zeta_{C}(s)$ sur $\mathbb{F}_{1}$ est la fonction de Riemann complète $\zeta_{\mathbb{Q}}(s)=\pi^{-s / 2} \Gamma(s / 2) \zeta(s)$. La fonction $N(q)$ est positive pour $q>1$ et donnée par

$$
N(q)=q-\frac{d}{d q}\left(\sum_{\rho \in Z} \operatorname{ordre}(\rho) \frac{q^{\rho+1}}{\rho+1}\right)+1
$$

où $Z$ est l'ensemble des zéros non-triviaux de la fonction zêta de Riemann et la dérivée est prise au sens des distributions. L'égalité (1) est une application typique des formules explicites de Riemann-Weil qui se formulent simplement sur le groupe des classes d'idèles. Il est donc naturel de rechercher à décrire, sinon la courbe $C$ elle même, du moins sa contrepartie par l'isomorphisme du corps de classes, comme un espace construit en termes adèliques et muni d'une action du groupe des classes d'idèles.

Nous construisons une compactification naturelle de l'espace $M=\mathbb{A}_{\mathbb{K}} / \mathbb{K}^{*}$ des classes d'adèles d'un corps global $\mathbb{K}$ en considérant $M$ comme un monoïde et en passant de la droite affine à l'espace projectif de dimension un, en utilisant le $\mathbb{F}_{1}$-schéma $\mathbb{P}_{\mathbb{F}}^{1}$. Nous montrons que la réalisation spectrale des zéros des fonctions $L$, l'équation fonctionnelle et les formules explicites apparaissent naturellement en calculant la cohomologie des faisceaux de fonctions sur $\mathbb{P}_{\mathbb{F}_{1}}^{1}(M)$. Plus précisément

(1) La cohomologie $H^{1}\left(\mathbb{P}_{\mathbb{F}_{1}}^{1}, \mathcal{F}\right)$ du faisceau $\mathcal{F}$ des fonctions complexes sur l'espace projectif $\mathbb{P}_{\mathbb{F}_{1}}^{1}(M)$ donne la réalisation spectrale des zéros des fonctions $L$ et la symétrie associée à l'équation fonctionnelle. 
(2) L'espace $\mathcal{P}(\mathbb{K})$ des éléments premiers du monoïde $M=\mathbb{A}_{\mathbb{K}} / \mathbb{K}^{*}$ sous l'action du groupe $C_{\mathbb{K}}$ est, en caractéristique $p>1$, isomorphe de manière équivariante à l'espace des valuations de l'extension abélienne maximale $\mathbb{K}^{\mathrm{ab}}$ munie de l'action du groupe de Weil : $\mathcal{W}^{\text {ab }} \subset \mathrm{Gal}\left(\mathbb{K}^{\mathrm{ab}}: \mathbb{K}\right)$.

(3) L'espace $\mathcal{P}(\mathbb{K})$ donne, en caractéristique zéro, une contrepartie de la courbe ${ }^{1}$ associée à un corps global en caractéristique $p>1$ et une interprétation des formules explicites de Riemann-Weil.

\section{WO-SCHÉMAS}

La théorie des $\mathfrak{W o - s c h e ́ m a s ~ s e ~ d e ́ v e ́ l o p p e ~ e n ~ p a r a l l e ̀ l e ~ a v e c ~ c e l l e ~ d e s ~ s c h e ́ m a s ~ e t ~}$ remonte à K. Kato, A. Deitmar, N. Kurokawa, H. Ochiai, M. Wakayama, B. TÖEn et M. VAQuiÉ.

On note $\mathfrak{W o}$ la catégorie des monoïdes commutatifs $M$ notés multiplicativement, avec élément neutre 1 et élément absorbant 0 (i.e. $0 \cdot x=0, \forall x \in M$ ). Un homomorphisme $\varphi: M \rightarrow N$ dans $\mathfrak{W o}$ est unifère (i.e. $\varphi(1)=1)$ et vérifie $\varphi(0)=0$. Un idéal $I$ d'un monoïde $M$ est un sous-ensemble $I \subset M$ tel que $0 \in I$ et:

$$
x \in I \Rightarrow x y \in I, \quad \forall y \in M .
$$

Un idéal $\mathfrak{p} \subset M$ est premier ssi son complément $\mathfrak{p}^{c}$ est multiplicatif i.e.

$$
x \notin \mathfrak{p}, y \notin \mathfrak{p} \Rightarrow x y \notin \mathfrak{p} .
$$

Le foncteur covariant

$$
\mathbb{F}_{1}[\cdot]: \mathfrak{A} \mathfrak{b} \rightarrow \mathfrak{M o} \quad H \mapsto \mathbb{F}_{1}[H]=H \cup\{0\}
$$

identifie la catégorie $\mathfrak{A} \mathfrak{b}$ des groupes abéliens à une sous-catégorie pleine de $\mathfrak{W} \mathfrak{o}$. Un espace monoïdal est un couple $\left(X, \mathcal{O}_{X}\right)$ où

- $X$ est un espace topologique ;

- $\mathcal{O}_{X}$ est un faisceau de monoïdes.

Les notions de morphismes, de spectre premier $\operatorname{Spec} M$, et de schéma géométrique, s'adaptent directement ( $c f$. [8]). La topologie de SpecM a pour base les ouverts

$$
D(f)=\{\mathfrak{p} \in \operatorname{Spec} M \mid f \notin \mathfrak{p}\}
$$

On appelle $\mathfrak{W} \mathfrak{o}$-foncteur tout foncteur covariant de la catégorie $\mathfrak{W} \mathfrak{o}$ vers la catégorie des ensembles. Un morphisme de $\mathfrak{W o - f o n c t e u r s , ~} \phi: X \rightarrow Y$ est une transformation naturelle, i.e. une application (ensembliste)

$$
\phi_{\mathrm{M}}: X(M) \rightarrow Y(M), \quad \forall M
$$

1. Plus précisément d'un revêtement abélien convenable. 
telle que pour tout homomorphisme $\rho: M \rightarrow M^{\prime}$ le diagramme suivant commute

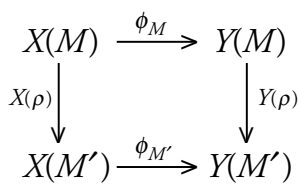

Un foncteur $\mathcal{F}$ est local ssi pour tout objet $M$ de $\mathfrak{W o}$ et recouvrement ouvert $\cup D\left(f_{j}\right)=\operatorname{Spec}(M)$ dans $M$ la suite suivante d'ensembles est exacte :

$$
\mathcal{F}(M) \stackrel{u}{\rightarrow} \prod_{i} \mathcal{F}\left(M_{f_{i}}\right) \rightarrow \prod_{i j} \mathcal{F}\left(M_{f_{i} f_{j}}\right)
$$

Lemme 2.1. Tout $\mathfrak{W o - f o n c t e u r ~ e s t ~ l o c a l . ~}$

Soit $Y$ un sous-foncteur du $\mathfrak{W o} \mathfrak{o}$-foncteur $X$, i.e. on a une inclusion $Y(M) \subset X(M)$ pour tout objet $M$ de $\mathfrak{W o}$.

On dit que $Y$ est ouvert dans $X$ ssi pour tout morphisme $\varphi: \operatorname{Spec}(M) \rightarrow X$ il existe un idéal $I \subset M$ tel que pour tout objet $N$ de $\mathfrak{W o}$ et tout $\rho \in \operatorname{Spec}(M)(N)=\operatorname{Hom}_{\mathfrak{W} \mathfrak{o}}(M, N)$ on ait :

$$
\varphi(\rho) \in Y(N) \subset X(N) \Leftrightarrow \rho(I) N=N .
$$

Soit $X$ un $\mathfrak{W o - f o n c t e u r . ~ O n ~ d i t ~ q u ' u n e ~ f a m i l l e ~} X_{\alpha}$ de sous-foncteurs de $X$ recouvre $X$ si pour tout groupe abélien $H$ l'ensemble $X\left(\mathbb{F}_{1}[H]\right)$ est la réunion des $X_{\alpha}\left(\mathbb{F}_{1}[H]\right)$. Pour $X=\operatorname{Spec} M$ et les $X_{\alpha}$ ouverts correspondants à des idéaux $I_{\alpha}$ la condition de recouvrement est équivalente à la condition ensembliste dans l'espace géométrique $\operatorname{Spec} M$, i.e. à $\exists \alpha, I_{\alpha}=M$. On prend $\varepsilon: M \rightarrow \mathbb{F}_{1}\left[M^{*}\right]=\kappa$, on a $\varepsilon \in X\left(\mathbb{F}_{1}[H]\right), H=M^{*} . \exists \alpha, \varepsilon \in X_{\alpha}\left(\mathbb{F}_{1}[H]\right)$ et donc $I_{\alpha} \cap M^{*} \neq \emptyset$ d'où $I_{\alpha}=M$.

Un $\mathfrak{W o}$-schéma est un $\mathfrak{W o}$-foncteur qui admet un recouvrement par des sousfoncteurs ouverts et affines.

Théorème 2.2. Tout $\mathfrak{W o - s c h e ́ m a ~} X$ est associé à un espace spectral (unique) $|X|$

$$
X(M)=\operatorname{Hom}(\operatorname{Spec} M,|X|), \quad \forall M
$$

\section{Collage de catégories et $\mathbb{F}_{1}$-Schémas}

On note $\mathfrak{A} \mathfrak{n}$ la catégorie des anneaux commutatifs unifères et

$$
\beta: \mathfrak{W o} \rightarrow \mathfrak{A n}, \quad M \mapsto \beta(M)=\mathbb{Z}[M]
$$

le foncteur qui associe à un monoïde $M$ l'anneau de convolution $\mathbb{Z}[M]$ (avec $0 \in M$ envoyé en 0 ), et $\beta^{*}$ le foncteur adjoint

$$
\beta^{*}: \mathfrak{A} \mathfrak{n} \rightarrow \mathfrak{W o}, \quad R \mapsto \beta^{*}(R)=R
$$

qui est le foncteur d'oubli de la structure additive. 
Un $\mathbb{F}_{1}$-schéma est donné par un $\mathfrak{W o - s c h e ́ m a ~} \underline{X}$, un $\mathbb{Z}$-schéma $X$ et une transformation naturelle de $\underline{X} \circ \beta^{*}$ vers $X$ qui est bijective lorsque $A=\mathbb{K}$ est un corps.

On peut de manière équivalente donner une transformation naturelle de $\underline{X}$ vers $X \circ \beta$.

Suivant une idée de P. Cartier on peut recoller deux catégories en utilisant un couple de foncteurs adjoints. On obtient la notion de $\mathbb{F}_{1}$-schéma en recollant la catégorie $\mathfrak{W o}$ avec celle $\mathfrak{A} \mathfrak{n}$ des anneaux commutatifs en utilisant le couple de foncteurs adjoints ci-dessus. On peut alors reformuler la notion de $\mathbb{F}_{1 \text {-schéma }}$ comme celle de foncteur covariant $X$ de $\mathfrak{W} \mathfrak{R}=\mathfrak{A} \mathfrak{n} \cup_{\beta, \beta^{*}} \mathfrak{W} \mathfrak{o}$ vers la catégorie des ensembles, tel que

— sa restriction à $\mathfrak{A} \mathfrak{n}$ est un $\mathbb{Z}$-schéma ;

— sa restriction à $\mathfrak{W o}$ est un $\mathfrak{W} o$-schéma ;

- la transformation naturelle associée à un corps est bijective.

Nous avons montré que les schémas sous-jacents aux groupes de Chevalley sont

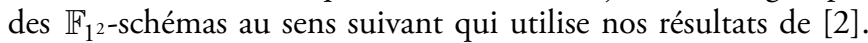

Théorème 3.1. Soit $\mathfrak{G}$ le schéma sur $\mathbb{Z}$ associé au groupe de Chevalley $G$.

- La construction du foncteur $\underline{G}$ se prolonge à la catégorie des couples $(M, \varepsilon)$ d'un monoïde abélien et un élément de carré 1.

- La construction de la transformation naturelle $e_{G}$ se prolonge aux anneaux commutatifs $A$ et donne une application

$$
e_{G, A}: \underline{G}(A,-1) \rightarrow \mathfrak{G}(A)
$$

- Quand $A$ est un corps l'application $e_{G, A}$ est une bijection.

\section{Cohomologie des faisceaux SUR UN WJo-schÉma}

Soit $X$ un espace topologique, la catégorie $\mathfrak{A} \mathfrak{b}(X)$ des faisceaux de groupes abéliens sur $X$ est une catégorie abélienne et elle a assez d'éléments injectifs [11]. Pour tout ouvert $U \subset X$ le foncteur de $\mathfrak{A} \mathfrak{b}(X)$ vers $\mathfrak{A} \mathfrak{b}$

$$
\mathcal{F} \mapsto \Gamma(\mathrm{U}, \mathcal{F})=\mathcal{F}(\mathrm{U})
$$

i.e. les sections de $\mathcal{F}$ sur $U$, est exact à gauche. Ses foncteurs dérivés sont les groupes de cohomologie $H^{P}(U, \mathcal{F})$. Pour $x \in X$ le foncteur de $\mathfrak{A} \mathfrak{b}(X)$ vers $\mathfrak{A} \mathfrak{b}$

$$
\mathcal{F} \mapsto \lim _{x \in U} \Gamma(U, \mathcal{F})=\mathcal{F}_{x}
$$


i.e. la fibre de $\mathcal{F}$ en $x$, est un foncteur exact. Pour tout ouvert $U \subset X$ de complémentaire $Y=U^{c}$, on a une longue suite exacte de cohomologie pour tout faisceau $\mathcal{F}$ de groupes abéliens :

$$
\begin{aligned}
0 & \mapsto H_{Y}^{0}(X, \mathcal{F}) \rightarrow H^{0}(X, \mathcal{F}) \rightarrow H^{0}\left(U,\left.\mathcal{F}\right|_{U}\right) \\
& \mapsto H_{Y}^{1}(X, \mathcal{F}) \rightarrow H^{1}(X, \mathcal{F}) \rightarrow H^{1}\left(U,\left.\mathcal{F}\right|_{U}\right) \\
& \mapsto H_{Y}^{2}(X, \mathcal{F}) \rightarrow \ldots
\end{aligned}
$$

où $H_{Y}$ désigne la cohomologie à support dans $Y$ et $\left.\mathcal{F}\right|_{U}$ est la restriction du faisceau $\mathcal{F}$ à l'ouvert $U$. Dans le cadre des $\mathfrak{W o}$-schémas ( $c f$. [8], [3]) on en déduit

Lemme 4.1. Soit $X$ la réalisation géométrique d'un $\mathfrak{W o - s c h e ́ m a . ~}$

1) Soit $U \subset X$ un ouvert affine, alors

$$
H^{p}(U, \mathcal{F})=0, \quad \forall p>0, \quad \mathcal{F} \in \text { obj } \mathfrak{A} \mathfrak{b}(X) .
$$

2) Soit $\mathcal{U}=\left\{U_{j}\right\}_{j \in J}$ un recouvrement ouvert de $X$ tel que les $\cap U_{j_{k}}$ soient affines, alors pour tout faisceau $\mathcal{F}$ sur $X$, on a l'égalité

$$
H^{p}(X, \mathcal{F})=\check{H}^{p}(\mathcal{U}, \mathcal{F}), \quad \forall p \geq 0
$$

avec la cohomologie de Čech associée au recouvrement $\mathcal{U}$.

3) Soit $Y=U^{c}$ le complémentaire d'un ouvert affine, on a, pour tout faisceau $\mathcal{F}$ de groupes abéliens, la suite exacte

$$
\begin{aligned}
0 & \mapsto H_{Y}^{0}(X, \mathcal{F}) \rightarrow H^{0}(X, \mathcal{F}) \rightarrow H^{0}\left(U,\left.\mathcal{F}\right|_{U}\right) \\
& \mapsto H_{Y}^{1}(X, \mathcal{F}) \rightarrow H^{1}(X, \mathcal{F}) \rightarrow 0
\end{aligned}
$$

\section{Espace Projectif des Classes D'AdèLes}

Nous renvoyons au $₫ 3$ pour la théorie des $\mathfrak{W} \mathfrak{o}$-schémas en tant que foncteurs covariants $M \rightarrow \underline{X}(M)$ de la catégorie $\mathfrak{W o}$ des monoïdes abéliens vers celle des ensembles. Soit $X$ l'espace géométrique associé. Nous utiliserons la projection canonique $\underline{X}(M) \rightarrow X$ ainsi définie

Lemme 5.1. Soit $\underline{X}$ un $\mathfrak{W o - s c h e ́ m a ~ e t ~} X$ sa réalisation géométrique. Pour tout monoïde $M$ il existe une application canonique

$$
\pi_{M}: \underline{X}(M) \rightarrow X, \pi_{M}(\phi)=\phi\left(\mathfrak{p}_{M}\right), \quad \forall \phi \in H_{m_{\mathfrak{W} \mathfrak{o}}}(\operatorname{Spec}(M), X)
$$

où $\mathfrak{p}_{M}$ est l'idéal premier maximal de $M$. Pour tout ouvert $U$ de $X$ on a

$$
\underline{U}(M)=\pi_{M}^{-1}(U) \subset \underline{X}(M)
$$

où $\underline{U}$ est le sous-foncteur de $\underline{X}$ associé à $U$. 
5.1. Le $\mathfrak{W o}$-schéma $\mathbb{P}_{\mathbb{F}}^{1}$. On prend le $\mathfrak{W o}$-schéma $\mathbb{P}_{\mathbb{F}_{1}}^{1}$. En tant que foncteur il est donné, pour tout monoïde $M$, en recollant deux copies de $M$ sur $M^{*}$ par $x \mapsto x^{-1}$,

$$
\underline{\mathbb{P}}_{\mathbb{F}_{1}}^{1}(M)=M \cup_{M^{*}} M
$$

Sa réalisation géométrique $(c f .[8],[3])$ i.e. l'espace topologique $\mathbb{P}_{\mathbb{F}_{1}}^{1}$ a trois points

$$
\mathbb{P}_{\mathbb{F}_{1}}^{1}=\{0, u, \infty\}, \overline{\{0\}}=\{0\}, \overline{\{u\}}=\mathbb{P}_{\mathbb{F}_{1}}^{1}, \overline{\{\infty\}}=\{\infty\}
$$

et trois ouverts $U_{ \pm}$et $U=U_{+} \cap U_{-}$dans $\mathbb{P}_{\mathbb{F}_{1}}^{1}$

$$
U_{+}=\mathbb{P}_{\mathbb{F}_{1}}^{1} \backslash\{\infty\}, U_{-}=\mathbb{P}_{\mathbb{F}_{1}}^{1} \backslash\{0\}, \quad U=U_{+} \cap U_{-}
$$

5.2. Le monoïde $M=\mathbb{A}_{\mathbb{K}} / \mathbb{K}^{*}$ des classes d'adèles. Soit $\mathbb{K}$ un corps global. Le groupe $C_{\mathbb{K}}$ des classes d'idèles est le groupe $M^{*}$ des éléments inversibles du monoïde

$$
M=\mathbb{A}_{\mathbb{K}} / \mathbb{K}^{*}, \quad \mathbb{K}^{*}=\mathrm{GL}_{1}(\mathbb{K})
$$

La projection canonique (10)

$$
\pi_{M}: \mathbb{P}_{\mathbb{F}_{1}}^{1}(M)=M \cup_{M *} M \rightarrow \mathbb{P}_{\mathbb{F}_{1}}^{1}
$$

associe $u \in \mathbb{P}_{\mathbb{F}_{1}}^{1}$ à tout élément de $M^{*}=C_{\mathbb{K}}$ et 0 ou $\infty$ aux autres éléments de $\underline{\mathbb{P}}_{\mathbb{F}_{1}}^{1}(M)$.

5.3. L'espace des fonctions sur $M$. Pour obtenir un espace naturel $\mathcal{S}(M)$ de fonctions sur $M$ on considère l'espace des coinvariants pour l'action de $\mathbb{K}^{*}$ donnée par $f_{q}(x)=f(q x)$ sur l'espace de Bruhat-Schwartz $\mathcal{S}\left(\mathbb{A}_{\mathbb{K}}\right)$ de fonctions à valeurs complexes. Plus précisément on part de la suite exacte

$$
0 \rightarrow \mathcal{S}\left(\mathbb{A}_{\mathbb{K}}\right)_{0} \rightarrow \mathcal{S}\left(\mathbb{A}_{\mathbb{K}}\right) \stackrel{\varepsilon}{\rightarrow} \mathbb{C} \oplus \mathbb{C}[1] \rightarrow 0
$$

où $\varepsilon(f)=\left(f(0), \int f(x) d x\right) \in \mathbb{C} \oplus \mathbb{C}[1]$ est $\mathbb{K}^{*}$-invariante et l'on pose

$$
\mathcal{S}(M)=\mathcal{S}_{0}(M) \oplus \mathbb{C} \oplus \mathbb{C}[1], \quad \mathcal{S}_{0}(M)=\mathcal{S}\left(\mathbb{A}_{\mathbb{K}}\right)_{0} / \overline{\left\{f-f_{q}\right\}}
$$

où $\overline{\left\{f-f_{q}\right\}}$ est la fermeture du sous-espace engendré par les $f-f_{q}, q \in \mathbb{K}^{*}$.

6. Le faisceau $\mathcal{F}$ SUR $\mathbb{P}_{\mathbb{F}_{1}}^{1}$ Des Demi-Densités Sur $\underline{\mathbb{P}}_{\mathbb{F}_{1}}^{1}(M)$

On définit un faisceau $\mathcal{F}$ sur $\mathbb{P}_{\mathbb{F}_{1}}^{1}$ en posant

$$
\begin{gathered}
\Gamma\left(U_{+}, \mathcal{F}\right)=\mathcal{S}(M) \\
\Gamma\left(U_{-}, \mathcal{F}\right)=\mathcal{S}(M) \\
\Gamma\left(U_{+} \cap U_{-}, \mathcal{F}=\mathcal{S}_{\infty}\left(C_{\mathbb{K}}\right)\right.
\end{gathered}
$$

où $\mathcal{S}_{\infty}\left(C_{\mathbb{K}}\right)$ est, pour les corps de nombres 


$$
\mathcal{S}_{\infty}\left(C_{\mathbb{K}}\right)=\cap_{\beta \in \mathbb{R}} \mu^{\beta} \mathcal{S}\left(C_{\mathbb{K}}\right)=\left\{f \in \mathcal{S}\left(C_{\mathbb{K}}\right) \mid \mu^{\beta} f \in \mathcal{S}\left(C_{\mathbb{K}}\right), \quad \forall \beta \in \mathbb{R}\right\},
$$

où $\mu \in C\left(C_{\mathbb{K}}\right)$ est le module $\mu: C_{\mathbb{K}} \rightarrow \mathbb{R}_{+}^{*}, \mu^{\beta}(g)=\mu(g)^{\beta}$ et $\mathcal{S}\left(C_{\mathbb{K}}\right)$ l'espace de Bruhat-Schwartz (cf. [7], [13]). Les applications de restriction à $U$ sont nulles sur les composantes $\mathbb{C} \oplus \mathbb{C}[1]$ et données par :

$$
\begin{aligned}
& (\operatorname{Res} f)(g)=\sum_{q \in \mathbb{K}^{*}} f(q g), \quad \forall f \in \mathcal{S}_{0}(M) \subset \Gamma\left(U_{+}, \mathcal{F}\right) \\
& (\operatorname{Res} h)(g)=|g|^{-1} \sum_{q \in \mathbb{K}^{*}} h\left(q g^{-1}\right), \quad \forall h \in \mathcal{S}_{0}(M) \subset \Gamma\left(U_{-}, \mathcal{F}\right) .
\end{aligned}
$$

6.1. Représentation de $N=C_{\mathbb{K}} \rtimes W$. Les égalités suivantes définissent une action de $C_{\mathbb{K}}$ sur le faisceau $\mathcal{F}$

$$
\begin{array}{ll}
\vartheta_{+}(\lambda) f(x)=f\left(\lambda^{-1} x\right), & \forall f \in \Gamma\left(U_{+}, \mathcal{F}\right) \\
\vartheta_{-}(\lambda) f(x)=|\lambda| f(\lambda x), & \forall f \in \Gamma\left(U_{-}, \mathcal{F}\right) \\
\vartheta(\lambda) f(x)=f\left(\lambda^{-1} x\right), & \forall f \in \Gamma\left(U_{+} \cap U_{-}, \mathcal{F}\right) .
\end{array}
$$

Le générateur $w=\left(\begin{array}{ll}0 & 1 \\ 1 & 0\end{array}\right)$ du groupe de Weyl $W$ de $\mathrm{PGL}_{2}$ agit sur $C_{\mathbb{K}}$ par l'automorphisme $g \mapsto g^{-1}$ de ce groupe abélien, ce qui donne un sens au produit semi-direct $N=C_{\mathbb{K}} \rtimes W$. Il agit sur $\mathbb{P}_{\mathbb{F}_{1}}^{1}$ en échangeant 0 et $\infty$. L'action de $w$ sur le faisceau $\mathcal{F}$ est donnée par :

$$
\begin{array}{llll}
w^{\#} f & = & f \in \Gamma\left(U_{-}, \mathcal{F}\right), & \forall f \in \Gamma\left(U_{+}, \mathcal{F}\right) \\
w^{\#} f & = & f \in \Gamma\left(U_{+}, \mathcal{F}\right), & \forall f \in \Gamma\left(U_{-}, \mathcal{F}\right) \\
w^{\#} f(g)= & |g|^{-1} f\left(g^{-1}\right), & \forall f \in \Gamma\left(U_{+} \cap U_{-}, \mathcal{F}\right) .
\end{array}
$$

qui envoie $\mathcal{F} \rightarrow w_{*} \mathcal{F}$.

Proposition 6.1. Il existe une unique action du produit semi-direct $N=C_{\mathbb{K}} \rtimes W$ sur le faisceau $\mathcal{F}$ donnée comme ci-dessus sur $W$ et sur $C_{\mathbb{K}}$ par la représentation $\vartheta\left[-\frac{1}{2}\right]$ obtenue comme produit tensoriel de $\vartheta$ par la représentation $\mu^{-1 / 2}$ de $C_{\mathbb{K}}$.

\section{RÉalisation Spectrale sur $H^{1}\left(\mathbb{P}_{\mathbb{F}_{1}}^{1}, \mathcal{F}\right)$}

7.1. Complexe de Čech. Le complexe de Čech du recouvrement $\mathcal{U}=\left\{U_{ \pm}\right\}$de $\mathbb{P}_{\mathbb{F}_{1}}^{1}$ a deux termes

$$
\begin{aligned}
C^{0} & =\Gamma\left(U_{+}, \mathcal{F}\right) \times \Gamma\left(U_{-}, \mathcal{F}\right) \\
C^{1} & =\Gamma\left(U_{+} \cap U_{-}, \mathcal{F}\right) .
\end{aligned}
$$

On introduit la notation $\sum(f)(x)=\sum_{\mathrm{q} \in \mathbb{K}^{*}} f(q x)$. Alors, le cobord $\partial: C^{0} \rightarrow C^{1}$ est donné par

$$
\partial(f, h)(g)=\sum(f)(g)-|g|^{-1} \sum(h)\left(g^{-1}\right) \in C^{1}
$$


Lemme 7.1. Le noyau de $\partial: C^{0} \rightarrow C^{1}$ est le graphe de la transformée de Fourier

$$
H^{0}\left(\mathbb{P}_{\mathbb{F}_{1}}^{1}, \mathcal{F}\right)=\left\{(f, F(f)) \mid f \in \mathcal{S}\left(\mathbb{A}_{\mathbb{K}}\right)_{0} / \overline{\left\{f-f_{q}\right\}} \oplus 2 \mathbb{C} \oplus 2 \mathbb{C}[1] .\right.
$$

Démonstration. Soit $\mathbb{K}$ un corps global, $\alpha$ un caractère non trivial de $\mathbb{A}_{\mathbb{K}} / \mathbb{K}$. Le réseau $\mathbb{K} \in \mathbb{A}_{\mathbb{K}}$ est son propre dual. La transformation de Fourier

$$
F(f)(a)=\int f(x) \alpha(a x) d x
$$

est définie canoniquement modulo le sous-espace $\left\{f-f_{q}\right\}$. La formule de Poisson s'écrit

$$
\sum h\left(g^{-1} q\right)=|g| \sum F(h)(g q)
$$

et donne pour $(f, h) \in \operatorname{Ker} \partial$

$$
\sum(F h)(g)=|g|^{-1} \sum(h)\left(g^{-1}\right) .
$$

D'où l'égalité

$$
\sum(f-F h)=0
$$

qui montre que $f-F h$ est nul dans l'espace des coinvariants [13].

Théorème 7.2. La représentation $\vartheta\left[-\frac{1}{2}\right]$ de $C_{\mathbb{K}}$ sur $H^{1}\left(\mathbb{P}_{\mathbb{F}_{1}}^{1}, \mathcal{F}\right)$ donne la réalisation spectrale des zéros des fonctions $L$. Cette représentation est invariante par la symétrie $\chi(g) \mapsto \chi\left(g^{-1}\right)$ du groupe des Grössencharakters du corps global $\mathbb{K}$.

Démonstration. On prend l'ouvert $U_{-} \subset \mathbb{P}_{\mathbb{F}_{1}}^{1}$ de complémentaire $Y=\{0\}$. La cohomologie à support $H_{Y}^{1}\left(\mathrm{U}_{+}, \mathcal{F}\right)$ donne la réalisation spectrale de [13], [4], [7], comme l'action de $C_{\mathbb{K}}$ sur le conoyau de l'application $\sum$. Par excision on a $H_{Y}^{1}\left(U_{+}, \mathcal{F}\right)=H_{Y}^{1}\left(\mathbb{P}_{\mathbb{F}_{1}}^{1}, \mathcal{F}\right)$ et comme $U_{-}$est affine, on a la suite exacte (9), d'où l'égalité

$$
0 \rightarrow H_{Y}^{1}\left(\mathbb{P}_{\mathbb{F}_{1}}^{1}, \mathcal{F}\right) \rightarrow H^{1}\left(\mathbb{P}_{\mathbb{F}_{1}}^{1}, \mathcal{F}\right) \rightarrow 0
$$

car

$$
H^{0}\left(\mathbb{P}_{\mathbb{F}_{1}}^{1}, \mathcal{F}\right) \rightarrow H^{0}\left(U_{-},\left.\mathcal{F}\right|_{U_{-}}\right)
$$

est surjective (Lemme 27).La symétrie résulte de l'action de $N=\mathrm{C}_{\mathbb{K}} \rtimes W$ sur le faisceau $\mathcal{F}$ et donc sur $H^{1}\left(\mathbb{P}_{\mathbb{F}_{1}}^{1}, \mathcal{F}\right)$.

8. Espace projectif des Classes D’adèles et $\mathbb{K}^{*} \backslash \mathrm{PGL}_{2}\left(\mathbb{A}_{\mathbb{K}}\right) / B\left(\mathbb{A}_{\mathbb{K}}\right)$

On a une inclusion naturelle

$$
\underline{P}_{\mathbb{F}_{1}}^{1}\left(\mathbb{A}_{\mathbb{K}} / \mathbb{K}^{*}\right) \subset \mathbb{K}^{*} \mid \mathrm{PGL}_{2}\left(\mathbb{A}_{\mathbb{K}}\right) / B\left(\mathbb{A}_{\mathbb{K}}\right)
$$


où l'on désigne par $B$ le sous-groupe de Borel des matrices triangulaires. On a en effet, avec $M=\mathbb{A}_{\mathbb{K}} / \mathbb{K}^{*}$,

$$
\underline{P}_{\mathbb{F}_{1}}^{1}(M)=M \cup_{M^{*}} M=\left(\mathbb{A}_{\mathbb{K}} \cup_{\mathbb{A}_{\mathbb{K}}^{*}} \mathbb{A}_{\mathbb{K}}\right) / \mathbb{K}^{*} \subset \mathbb{P}_{\mathbb{Z}}^{1}\left(\mathbb{A}_{\mathbb{K}}\right) / \mathbb{K}^{*}
$$

Soit $\mathcal{T}$ le tore maximal, et $\mathcal{N}$ son normalisateur. On a

$$
\mathbb{K}^{*}=\mathcal{T}(\mathbb{K}) \subset \mathcal{T}\left(\mathbb{A}_{\mathbb{K}}\right), \quad \mathcal{T}\left(\mathbb{A}_{\mathbb{K}}\right) / \mathcal{T}(\mathbb{K}) \sim C_{\mathbb{K}}, \quad \mathcal{N}\left(\mathbb{A}_{\mathbb{K}}\right) / \mathcal{T}(\mathbb{K}) \sim C_{\mathbb{K}} \rtimes W
$$

Le quotient de $\mathrm{PGL}_{2}\left(\mathbb{A}_{\mathbb{K}}\right) / B\left(\mathbb{A}_{\mathbb{K}}\right)$ par $\mathbb{K}^{*}$ est identique à l'espace des feuilles du feuilletage de $\mathcal{T}(\mathbb{K}) \backslash \mathrm{PGL}_{2}\left(\mathbb{A}_{\mathbb{K}}\right)$ par les orbites de l'action de $B\left(\mathbb{A}_{\mathbb{K}}\right)$. Le faisceau $\mathcal{F}$ est celui des demi-densités. L'interprétation donnée par Guillemin dans [12] de la formule des traces de Selberg en termes de la quantification géométrique associée au feuilletage horocyclique, est analogue à l'interprétation ci-dessous (cf. \$10) des formules explicites.

\section{L'action de $C_{\mathbb{K}}$ SUR Les ÉlÉments PRemiers de $M=\mathbb{A}_{\mathbb{K}} / \mathbb{K}^{*}$}

Soit $\mathbb{K}$ un corps global de caractéristique $p>1$ et $\mathbb{F}_{q} \subset \mathbb{K}$ son corps de constantes. Les points de la courbe $C$ sur $\mathbb{F}_{q}$, projective et non-singulière dont $\mathbb{K}$ est le corps de fonctions sont les valuations discrètes du corps

$$
\mathbb{K}^{\mathrm{ar}}=\overline{\mathbb{F}}_{q} \otimes_{\mathbb{F} q} \mathbb{K} .
$$

Considérons, au lieu de $\mathbb{K}^{\text {ar }}$, l'extension abélienne maximale (séparable) $\Omega=\mathbb{K}^{\mathrm{ab}}$ de $\mathbb{K}$. Nous montrons que l'action du groupe de Weil abélianisé $\mathcal{W}^{\mathrm{ab}} \subset \mathrm{Gal}\left(\mathbb{K}^{\mathrm{ab}}: \mathbb{K}\right)$ sur l'espace des valuations de $\mathbb{K}^{\text {ab }}$ est isomorphe à l'action du groupe $C_{\mathbb{K}}$ des classes d'idèles sur l'espace $\mathcal{P}(\mathbb{K})$ des éléments premiers du monoïde $M=\mathbb{A}_{\mathbb{K}} / \mathbb{K}^{*}$. Nous dirons qu'un élément $p \in M$ d'un monoïde $M$ est premier si l'idéal $p M$ qu'il engendre est premier (i.e. son complémentaire est multiplicatif). On identifie le groupe $\mathcal{W}^{\mathrm{ab}} \subset \mathrm{Gal}\left(\mathbb{K}^{\mathrm{ab}}: \mathbb{K}\right)$ avec $C_{\mathbb{K}}$ par la théorie du corps de classe.

Proposition 9.1. L'espace $\mathrm{val}\left(\mathbb{K}^{\mathrm{ab}}\right)$ des valuations de $\mathbb{K}^{\mathrm{ab}}$ muni de l'action de $\mathcal{W}^{\mathrm{ab}} \subset \mathrm{Gal}\left(\mathbb{K}^{\mathrm{ab}}: \mathbb{K}\right)$ est isomorphe de manière équivariante non-canonique à l'espace $\mathcal{P}(\mathbb{K})$ des éléments premiers du monö̈de $M=\mathbb{A}_{\mathbb{K}} / \mathbb{K}^{*}$ sous l'action du groupe $C_{\mathbb{K}}$.

Tout idéal premier principal du monoïde $M=\mathbb{A}_{\mathbb{K}} / \mathbb{K}^{*}$ est de la forme

$$
\mathfrak{p}_{v}=\left\{x \in M \mid x_{v}=0\right\}
$$

où $v \in \sum_{\mathbb{K}}$ est une place de $\mathbb{K}$. Pour les deux espaces val $\left(\mathbb{K}^{\mathrm{ab}}\right)$ et $\mathcal{P}(\mathbb{K})$ on a une application canonique vers l'ensemble des places de $\mathbb{K}$

$$
\operatorname{val}\left(\mathbb{K}^{\mathrm{ab}}\right) \rightarrow \sum_{\mathbb{K}} \leftarrow \mathcal{P}(\mathbb{K})
$$

et l'on montre que les groupes d'isotropie se correspondent par l'isomorphisme $\mathcal{W}^{\mathrm{ab}} \sim C_{\mathbb{K}}$ de la théorie du corps de classes. 
En caractéristique zéro, il manque un morceau Galoisien dans l'isomorphisme du corps de classe, mais l'espace $\mathcal{P}(\mathbb{K})$ des éléments premiers du monoïde $M=\mathbb{A}_{\mathbb{K}} / \mathbb{K}^{*}$ sous l'action du groupe $C_{\mathbb{K}}$ continue à avoir un sens. Une motivation importante de l'étude de $\overline{\mathbb{F}}_{1}(c f$. [6]) est d'arriver à donner à l'espace $\mathcal{P}(\mathbb{K})$ une structure géométrique qui joue un rôle analogue à celle de la courbe $C$.

\section{io. Orbites périodiques SUR $\mathbb{P}^{1}$}

Le passage de l'espace des classes d'adèles $M$ à l'espace projectif $\mathbb{P}_{\mathbb{F}_{1}}(M)$ double le nombre des orbites périodiques de l'action de $C_{\mathbb{K}}$. Nous vérifions ci-dessous que le même doublement apparaît dans le calcul de $H^{0}-H^{1}$ pour l'espace projectif, quand on passe de la cohomologie à support $H_{Y}$ à la cohomologie globale. Pour $(x, u) \in M \times C_{\mathbb{K}}$, tel que $u x=x$, avec $u \neq 1$, il existe une place $v \in \sum_{\mathbb{K}}$ telle que $x \in \mathfrak{p}_{v}$. Lespace transverse à $\mathfrak{p}_{v} \subset M$ s'identifie au corps local $\mathbb{K}_{v}$. L'action du groupe d'isotropie $I_{v}=\mathbb{K}_{v}^{*}$ est l'action de $\mathbb{K}_{v}^{*}$ sur $\mathbb{K}_{v}$ par multiplication. La trace au sens des distributions est donnée par (cf. [7])

$$
\operatorname{Tr}_{\text {distr }}\left(\int h(u) \vartheta(u) d^{*} u\right)=\sum_{v} \int_{\mathbb{K}_{v}^{*}} \frac{h(u-1}{|1-u|} d^{*} u
$$

On note succintement $\int_{\mathbb{K}_{v}^{*}}^{\prime}$ la contribution locale $\int_{\mathbb{K}_{v}^{*}} \frac{b(u-1)}{|1-u|} d^{*} u$. Les formules explicites de Weil s'écrivent

$$
\tau+\tau[1]-\sum \tau[\chi, \rho]=\sum \int_{\mathbb{K}_{v}^{*}}^{\prime}
$$

où $\tau$ et $\tau[1]$ désignent les caractères de la représentation triviale de $C_{\mathbb{K}}$ et $\mathrm{du}$ module $\mu$, et $\tau[\chi, \rho]$ le caractère associé à un zéro $\rho \in Z_{\chi}$ de la fonction $L(\chi, s)$. Pour la cohomologie à support (26) on a directement

$$
\begin{gathered}
H_{Y}^{0}\left(\mathbb{P}_{\mathbb{F}_{1}}^{1}, \mathcal{F}\right)=\tau+\tau[1] \\
H_{Y}^{1}\left(\mathbb{P}_{\mathbb{F}_{1}}^{1}, \mathcal{F}\right)=H^{1}\left(\mathbb{P}_{\mathbb{F}_{1}}^{1}, \mathcal{F}\right)=\sum \tau[\chi, \rho]=\tau+\tau[1]-\sum \int_{\mathbb{K}_{v}^{*}}^{\prime}
\end{gathered}
$$

et l'on obtient

$$
H_{Y}^{0}\left(\mathbb{P}_{\mathbb{F}_{1}}^{1}, \mathcal{F}\right)-H_{Y}^{1}\left(\mathbb{P}_{\mathbb{F}_{1}}^{1}, \mathcal{F}\right)=\sum \int_{\mathbb{K}_{v}^{*}}^{\prime}
$$

Le passage à l'espace projectif double le nombre d'orbites périodiques de l'action de $C_{\mathbb{K}}$ et il faut vérifier que ceci est cohérent avec le calcul de $H^{0}$ et $H^{1}$ pour $\mathbb{P}^{1}$. On a en effet, en notant $\Lambda$ la représentation régulière de $C_{\mathbb{K}}$,

$$
\begin{gathered}
H^{0}\left(\mathbb{P}_{\mathbb{F}_{1}}^{1}, \mathcal{F}\right)=\Lambda+\sum \int_{\mathbb{K}_{v}^{*}}^{\prime}+\tau+\tau[1] \\
H^{1}\left(\mathbb{P}_{\mathbb{F}_{1}}^{1}, \mathcal{F}\right)=\sum \tau[\chi, \rho]=\tau+\tau[1]-\sum \int_{\mathbb{K}_{v}^{*}}^{\prime} \\
H^{0}\left(\mathbb{P}_{\mathbb{F}_{1}}^{1}, \mathcal{F}\right)-H^{1}\left(\mathbb{P}_{\mathbb{F}_{1}}^{1}, \mathcal{F}\right)=\Lambda+2 \sum \int_{\mathbb{K}_{v}^{*}}^{\prime}
\end{gathered}
$$

ce qui rend bien compte du doublement du nombre d'orbites périodiques de l'action de $C_{\mathbb{K}}$. 


\section{CONFÉRENCES}

Septembre 2008, 1 conférence à Muenster (en l'honneur de Joachim Cuntz).

Octobre 2008, 2 conférences à Rome (avec Colloquium en physique).

Novembre 2008, 1 conférence à Munich (Colloquium en physique).

Mars 2009, 1 conférence à Lausanne (en l'honneur de Ludwig Faddeev).

Mars 2009, 1 conférence à Johns Hopkins (à l'occasion de la rencontre JAMI).

Mai 2009, 5 conférences à Vanderbilt (Fifth Spring Institute in Noncommutative Geometry and Operator Algebras).

Juin 2009, 1 conférence à Unesco, Paris (Invisible universe).

Juillet 2009, 1 conférence à Bonn (en l'honneur d'Henri Moscovici).

Juin 2009, 1 conférence à Unesco, Paris (XII e congrès Marcel Grossmann).

\section{Publications}

A. Connes, A unitary invariant in Riemannian Geometry, Dédié à Michel DuboisViolette.

A. Connes, C. Consani, On the notion of geometry over $\mathbb{F}_{1}$ (preprint) arXiv : 0809.2926v2 [mathAG]. Journal of Algebraic Geometry.

A. Connes, C. Consani, Schemes over $\mathbb{F}_{1}$ and zeta functions (preprint) arXiv : 0903.2024v2 [mathAG].

\section{RÉFÉRENCES}

[1] A. Connes, Trace formula in noncommutative geometry and the zeros of the Riemann zeta function. Selecta Math. (N.S.) 5 (1999), nº 1, 29-106.

[2] A. Connes, C. Consani, On the notion of geometry over $\mathbb{F}_{1}$ (preprint) arXiv: 0809.2926v2 [mathAG]. To appear in Journal of Algebraic Geometry.

[3] A. Connes, C. Consani, Schemes over $\mathbb{F}_{1}$ and zeta functions (preprint) arXiv: 0903.2024v2 [mathAG].

[4] A. Connes, C. Consani, M. Marcolli, Noncommutative geometry and motives: the thermodynamics of endomotives, Advances in Mathematics, vol. 214 (2007) $\mathrm{n}^{\circ}$ 2, 761-831.

[5] A. Connes, C. Consani, M. Marcolli, The Weil proof and the geometry of the adeles class space, to appear in "Algebra, Arithmetic and Geometry - Manin Festschrift ", Progress in Mathematics, Birkhäuser(2008); preprint arXiv0703.392.

[6] A. Connes, C. Consani, M. Marcolli, Fun with $\mathbb{F}_{1}$, Journal of Number Theory 129 (2009) p. 1532-1561.

[7] A. Connes, M. Marcolli, Noncommutative Geometry, Quantum Fields, and Motives, Colloquium Publications, vol. 55, American Mathematical Society, 2008.

[8] A. Deitmar, Schemes over F1, in Number Fields and Function Fields - Two Parallel Worlds. Ed. by G. van der Geer, B. Moonen, R. Schoof. Progr. in Math, vol. 239, 2005.

[9] A. Deitmar, F1-schemes and toric varieties, Contributions to Algebra and Geometry vol. 49, $\mathrm{n}^{\circ} 2$, pp. 517-525 (2008).

[10] A. Deitmar, Remarks on zeta functions and K-theory over F1. Proc. Japan Acad. Ser. A Math. Sci. $82 \mathrm{n}^{\circ}$ 8, 141-146 (2006).

[11] A. Grothendieck, Sur quelques points d'algèbre homologique Tohoku Math. J., t. 9, 1957, p. 119-183.

[12] V. Guillemin, Lectures on spectral theory of elliptic operators, Duke Math. J., vol. 44, 3 (1977), 485-517.

[13] R. Meyer, On a representation of the idele class group related to primes and zeros of L-functions. Duke Math. J., vol. 127 (2005), nº3, 519-595.

[14] C. Soulé, Les variétés sur le corps à un élément. Mosc. Math. J. 4 (2004), nº 1, $217-$ 244, 312.

[15] A. Weil, Sur la théorie du corps de classes, J. math. Soc. Japan, t. 3, 1951, p. 1-35. 